\title{
Influência da vitamina D nas doenças endocrinometabólicas
}

\section{Influence of vitamin D in endocrine metabolic diseases}

\author{
Rafael Algusto Rafaelli ${ }^{1}$; Priscila Ruzzon Nomura²; Fernanda Dias Figueira ${ }^{3}$; \\ Isabela Cristina Pinheiro de Freitas Santos ${ }^{4}$; Lorena Flor da Rosa Santos Silva ${ }^{5}$; \\ Danielle Venturini ${ }^{6}$
}

\begin{abstract}
Resumo
A deficiência de vitamina $\mathrm{D}$ tem sido relacionada com o desenvolvimento de diversas doenças endocrinometabólicas, como por exemplo, síndrome metabólica, obesidade, hipertensão e diabetes mellitus tipo 2. Este trabalho apresenta uma visão geral sobre as evidências científicas disponíveis para algumas das ações não-calcêmica da vitamina D nos seres humanos, através de pesquisa bibliográfica em bases de dados científicos. A deficiência de vitamina $\mathrm{D}$ pode predispor à intolerância à glicose, a alterações na secreção de insulina e, assim, ao desenvolvimento de diabetes mellitus tipo 2. Esse possível mecanismo ocorre em razão da presença do receptor de vitamina $\mathrm{D}$ em diversas células e tecidos, incluindo células $\beta$ do pâncreas, no adipócito e no tecido muscular. Em indivíduos obesos, as alterações do sistema endócrino da vitamina $\mathrm{D}$, caracterizada por elevados níveis de paratormônio e da 1,25-dihidroxicolecalciferol são responsáveis pelo feedback negativo da síntese hepática de 25-hidroxicolecalciferol e também pelo maior influxo de cálcio para o meio intracelular, que pode prejudicar a secreção e a sensibilidade à insulina. Na hipertensão, a vitamina $\mathrm{D}$ pode atuar via sistema renina-angiotensina e também na função vascular. Há evidências de que a 1,25-dihidroxicolecalciferol inibe a expressão da renina e bloqueia a proliferação da célula vascular muscular lisa. São necessários mais estudos prospectivos e ensaios clínicos randomizados, incluindo estudos de suplementação, que estabeleçam melhor os efeitos clínicos e metabólicos das variações na concentração de 25-hidroxicolecalciferol na evolução clínica dessas doenças.
\end{abstract}

Palavras-chave: Vitamina D. Síndrome metabólica. Diabetes Mellitus. Hipertensão. Obesidade.

\begin{abstract}
The vitamin D deficiency has been linked to the development of several endocrine metabolic diseases such as metabolic syndrome, obesity, hypertension and Type 2 diabetes mellitus. This paper presents an overview of the available scientific evidence for some of the non- calcemic actions of vitamin D in humans, through literature search in scientific databases. The deficiency of vitamin D may predispose to glucose intolerance, changes in insulin secretion and thus the development of type 2 diabetes mellitus. This mechanism is
\end{abstract}

${ }^{1}$ Farmacêutico, Residente em Análises Clínicas, Departamento de Patologia, Análises Clínicas e Toxicológicas, Universidade Estadual de Londrina (UEL), Londrina-PR.

${ }^{2}$ Farmacêutica, Residente em Análises Clínicas, Departamento de Patologia, Análises Clínicas e Toxicológicas, Universidade Estadual de Londrina (UEL), Londrina-PR.

${ }^{3}$ Farmacêutica, Residente em Análises Clínicas, Departamento de Patologia, Análises Clínicas e Toxicológicas, Universidade Estadual de Londrina (UEL), Londrina-PR.

${ }^{4}$ Farmacêutica, Residente em Análises Clínicas, Departamento de Patologia, Análises Clínicas e Toxicológicas, Universidade Estadual de Londrina (UEL), Londrina-PR.

${ }^{5}$ Farmacêutica, Residente em Análises Clínicas, Departamento de Patologia, Análises Clínicas e Toxicológicas, Universidade Estadual de Londrina (UEL), Londrina-PR.

${ }^{6}$ Farmacêutica-bioquímica, Doutora em Ciências da Saúde, Professora do Centro de Ciências da Saúde, Departamento de Patologia, Análises Clínicas e Toxicológicas, área Bioquímica Clínica, Universidade Estadual de Londrina (UEL), Londrina-PR. 
possible due to the presence of the vitamin $\mathrm{D}$ receptor in several tissues and cells, including pancreatic $\beta$ cells, adipocyte and muscle tissue. In obese individuals, the changes of the vitamin D endocrine system, characterized by high levels of parathyroid hormone and 1,25-dihydroxycholecalciferol are responsible for the negative feedback of hepatic synthesis of 25-hydroxycholecalciferol and also by increased influx of calcium into the intracellular environment, which can damage the secretion and insulin sensitivity. In hypertension, vitamin D could act on the renin-angiotensin system and also in vascular function. There is some evidence that 1,25-dihydroxycholecalciferol inhibits the renin gene expression and blocks the proliferation of vascular smooth muscle cell. Further prospective studies and randomized clinical trials, including studies of supplementation, are required to establish better clinical and metabolic effects of variations in the concentration of 25-hydroxycholecalciferol in the clinical course of these diseases.

Keywords: Vitamin D. Metabolic syndrome. Diabetes Mellitus. Hypertension. Obesity.

\section{Introdução}

A vitamina $\mathrm{D}$ é um fator indispensável para o desenvolvimento e manutenção do tecido ósseo, bem como para a manutenção da homeostase do cálcio e do fósforo. Atualmente, várias evidências sugerem que esse composto desempenhe outras funções além dessa, como por exemplo, diferenciação e proliferação celular, secreção hormonal, e também atuando no sistema imune e em diversas doenças crônicas não transmissíveis, como por exemplo, diabetes mellitus tipo 2, hipertensão arterial e obesidade.

De acordo com Holick (2004) são considerados quadros de deficiência níveis de 25-hdroxicolecalciferol - 25(OH)D abaixo de 20 $\mathrm{ng} / \mathrm{mL}$, e de insuficiência, abaixo de $32 \mathrm{ng} / \mathrm{mL}$, no entanto não há um consenso quanto a esses valores, e alguns autores propõem níveis individualizados de acordo com idade e sexo.

Em 2010, o Institute of Medicine (IOM, 2011), publicou novas recomendações para a ingestão dietética de vitamina $\mathrm{D}$, justificando que os novos valores de referência foram baseados em estudos publicados em revistas de grande importância no meio científico, sobre a relevância da vitamina D. A vitamina $\mathrm{D}$ possui múltiplas funções fisiológicas além do seu papel clássico no metabolismo ósseo e homeostase do cálcio. Há evidências de estudos epidemiológicos com a vitamina $\mathrm{D}$, ensaios clínicos de intervenção, bem como estudos utilizando animais, em que o comprometimento no status da vitamina $\mathrm{D}$ e do cálcio advindos da alimentação inadequada é um fator predisponente para diversas patologias, incluindo vários tipos de câncer, doenças infecciosas, inflamatórias e autoimunes, cardiovasculares e metabólicas, como obesidade e hipertensão.

Estima-se que receptores de vitamina D (Vitamin D receptor-VDR) estão envolvidos na expressão de aproximadamente 3.000 genes humanos (FORD et al., 2005; LIU et al., 2005; SCHUCH et al., 2009). Atualmente, ahipovitaminoseD tem sidoconsiderada um problema de saúde pública mundial, devido a sua implicação no desenvolvimento de doenças metabólicas e aumento de risco cardiovascular. Portanto, o presente trabalho teve como objetivo realizar uma revisão bibliográfica sobre a vitamina $\mathrm{D}$ e a influência da hipovitaminose D no aparecimento de algumas doenças endocrinometabólicas, dentre elas: síndrome metabólica (SM), diabetes mellitus tipo 2 (DM2), hipertensão arterial (HA) e obesidade.

\section{Material e Métodos}

A metodologia foi baseada em uma pesquisa bibliográfica em bases de dados científicos: LILACS, MEDLINE, Pubmed e SciELO, utilizando os seguintes termos: Vitamina D (Vitamin D), Síndrome Metabólica e Vitamina D (Metabolic Syndrome and Vitamin D), Hipertensão e Vitamina D (Hypertension and Vitamin D), Obesidade e Vitamina D (Obesity and Vitamin D), Diabetes Mellitus e Vitamina D (Diabetes Mellitus and Vitamin D). 


\section{Revisão de Literatura}

\section{Vitamina D e Síndrome Metabólica}

A síndrome metabólica (SM) pode ser entendida com um grupo de fatores de risco que estão interrelacionados, de origem metabólica, que podem contribuir diretamente para o desenvolvimento de doenças cardiovasculares (DCV) e diabetes mellitus do tipo 2 entre outras patologias. São considerados fatores de risco metabólicos segundo Grundy et al. (2005): dislipidemia aterogênica (hipertrigliceridemia, níveis elevados de apolipoproteína B, partículas de LDL-colesterol pequenas e densas e níveis baixos de HDL- colesterol), hipertensão arterial, hiperglicemia e um estado pró-inflamatório e pró-trombótico, que é encontrado muitas vezes no indivíduo obeso.

Os critérios utilizados pela National Cholesterol Education Program (NCEP) norte-americano, que descrevem os componentes da síndrome de maneira a facilitar o seu diagnóstico, em um trabalho conhecido como ATP III (Adult Treatment Panel), são os mais amplamente utilizados no diagnóstico da SM, tanto na prática clínica como em estudos epidemiológicos. No entanto, existem outros critérios entre eles o da International Diabetes Federation (IDF) e o da Organização Mundial da Saúde (OMS). Os critérios da NCEP/ATP III se encontram na tabela a seguir:

Tabela 1 - Definição de SM segundo Adult Treatment Panel III (ATP III)

\begin{tabular}{|l|l|}
\hline \multicolumn{1}{|c|}{ Parâmetro } & \multicolumn{1}{c|}{$\begin{array}{c}\text { Devem estar presentes pelo menos três dos componentes } \\
\text { abaixo: }\end{array}$} \\
\hline Glicose & $\geq 100 \mathrm{mg} / \mathrm{dL}$ ou em tratamento para hiperglicemia \\
\hline HDL-colesterol & $\begin{array}{l}\text { Homens: }<40 \mathrm{mg} / \mathrm{dL} \text { ou em tratamento para HDL baixo } \\
\text { Mulheres: }<50 \mathrm{mg} / \mathrm{dL} \text { ou em tratamento para HDL baixo }\end{array}$ \\
\hline Triacilglicerol & $\geq 150 \mathrm{mg} / \mathrm{dL} \mathrm{ou} \mathrm{em} \mathrm{tratamento} \mathrm{para} \mathrm{hipertrigliceridemia}$ \\
\hline Circunferência abdominal & Cintura $\geq 102 \mathrm{~cm}$ para homens ou $\geq 88 \mathrm{~cm}$ para mulheres \\
\hline Hipertensão (HAS) & $\geq 130 \times 85 \mathrm{mmHg}$ ou em tratamento medicamentoso para HAS \\
\hline
\end{tabular}

Fonte: Grundy et al. (2005)

Não está estabelecida ainda a causa ou as múltiplas causas da SM, porém é sabido que a obesidade abdominal e a resistência à insulina parecem ter um papel fundamental na gênese desta síndrome. Achados epidemiológicos também sugerem que baixos níveis séricos de $25(\mathrm{OH})$ $\mathrm{D}$ estão associados com o aumento do risco de desenvolver a SM.

Ford et al. (2005), utilizando-se de uma amostragem representativa da população dos Estados Unidos que participaram da National Health and Nutrition Examination Survey (NHANES III), relataram uma associação entre o status de vitamina D e SM em 8.421 homens e mulheres não grávidas que tinham 20 anos de idade ou mais. Para investigar a associação entre vitamina D e a SM, a amostra foi dividida com base em quintis de concentrações séricas de 25(OH)D para determinar se a porção da população com SM diferia por status de vitamina D e para testar uma tendência linear entre essas variáveis. Como esperado, um em cada cinco indivíduos adultos tinha SM. A concentração sérica média de 25(OH)D nos pacientes com SM foi de $67,1 \mathrm{nmol} / \mathrm{L}$, significativamente menor do que nos pacientes sem SM, com uma média de $75,9 \mathrm{nmol} / \mathrm{L}$. O estudo também encontrou uma associação inversa significativa para quintis de níveis séricos de 25-hidroxivitamina $\mathrm{D}$ com alguns dos componentes individuais da SM, incluindo obesidade abdominal, hipertrigliceridemia e hiperglicemia.

No mesmo ano, Liu et al. (2005) analisaram dados de 10.066 mulheres, com idade de 45 anos ou mais que participaram do Women 's Health Study para avaliar a ingestão dietética de vitamina D e cálcio e sua associação com a prevalência de SM. 
Os dados foram analisados por quintis de cálcio total e ingestão de vitamina $\mathrm{D}$; um modelo de regressão logística foi aplicado para examinar a associação entre cálcio e ingestão de vitamina D e o risco de SM. O consumo médio de cálcio nesta coorte foi de 857 $\mathrm{mg} /$ dia e a ingestão média de vitamina $\mathrm{D}$ foi de 266 $\mathrm{UI} / \mathrm{d}$ (6,65 g/dia). A prevalência de componentes da SM foi menor em mulheres no quintil mais alto de cálcio combinado com a vitamina $\mathrm{D}$ do que naqueles quintis com menor ingestão, embora não seja possível afirmar que o efeito observado foi devido a atuação exclusiva da vitamina $\mathrm{D}$.

Recentemente, Miñambres et al. (2012) conduziram um estudo transversal de 343 indivíduos obesos ou com sobrepeso. Foram analisados os dados antropométricos e a presença ou ausência de SM. Adicionalmente foram determinadas as concentrações de $25(\mathrm{OH}) \mathrm{D}$, insulina e o índice Homeostasis Model Assessment insulin resistance (HOMA-IR). Quarenta e seis pacientes $(13,4 \%)$ apresentavam os critérios de SM. A vitamina D foi associada com o grau de obesidade, especialmente quando o índice de massa corporal (IMC) era $>40$ $\mathrm{kg} / \mathrm{m} 2$. Pacientes com SM tinham níveis mais baixos de $25(\mathrm{OH}) \mathrm{D}$ do que os pacientes sem SM (43,3 \pm 29,0 vs $55,3 \pm 29,6 \mathrm{mmol} / \mathrm{L}$, respectivamente) e a razão de chance para hipovitaminose $\mathrm{D}$ foi de 2,7 (intervalo de confiança (IC), 1,14-6,4 ( $\mathrm{p}=$ $0,023)$ em pacientes com SM versus pacientes sem $\mathrm{SM}$, independentemente do grau de obesidade. Os dados deste estudo confirmam a associação entre a vitamina D e SM e sugerem que esta associação seja independente do grau de obesidade.

Outro estudo transversal, conduzido por Kayaniyil et al. (2011) examinou a associação da vitamina D e paratormônio (PTH) com a SM e seus componentes em uma grande amostra multiétnica. Foram examinados 654 participantes de Londres, Toronto, Ontário e Canadá com idade de 30 anos ou mais, e que apresentavam fatores de risco para diabetes mellitus tipo 2. Foi verificada a presença ou ausência de SM e seus componentes. O PTH não foi associado com a presença de SM, após ajustes multivariados. Os dados mostraram associação inversa de concentrações séricas de 25(OH)D com a circunferência da cintura, o nível sérico de triacilglicerol, insulina de jejum, e alanina aminotransaminase $(\mathrm{p}<0,041)$. Níveis elevados de PTH foram associados positivamente com a circunferência da cintura e lipoproteína de alta densidade-colesterol (HDL-c) $(\mathrm{p}<0,04)$.

Existem vários outros estudos transversais que corroboram com os dados apresentados por estes acima citados. No entanto, estudos prospectivos têm sido limitados e assim, nenhuma relação de causa e efeito pode ser inferida. Al-Daghri et al. (2012), realizaram um estudo de intervenção com um ano de duração onde foram verificadas reversões modestas nos componentes da SM, por meio da correção do status de vitamina D. Um total de 59 adultos árabes (31 homens e 28 mulheres) não diabéticos, com sobrepeso ou obesos, foram prospectivamente envolvidos neste estudo intervencionista de um ano. Na visita inicial (dados basais), a prevalência de HDLcolesterol baixo e hipertensão foi significativamente maior entre os pacientes com deficiência de vitamina $\mathrm{D}(\mathrm{p}<0,05)$, mesmo após o ajuste para sexo e IMC. A prevalência geral de SM nos participantes, pelos critérios do NCEP/ATPIII, diminuiu de 25,2\% para $13,0 \%$ no final do período analisado.

\section{Vitamina D e Diabetes Mellitus}

O diabetes mellitus (DM) constitui um grupo de doenças no qual as concentrações plasmáticas de glicose encontram-se elevadas, como resultado da deficiência na secreção de insulina pelo pâncreas e/ ou ação inadequada da insulina. A hiperglicemia ocasionada por essa situação provoca danos a longo prazo, disfunção e falência de vários órgãos, principalmente olhos, rins, nervos, coração e vasos sanguíneos (SOCIEDADE BRASILEIRA DE DIABETES, 2006).

A doença constitui um problema de saúde pública, devido à elevada prevalência, morbimortalidade $\mathrm{e}$ custos do tratamento. Segundo Malerbil e Franco (1992, p. 1510) “[...] Estima-se que em 2030 haverá 
um acréscimo de $42 \%$ no número de indivíduos com DM no mundo. No Brasil, a prevalência total do DM é de 7,6\% e destes, $46 \%$ desconhecem ter o diagnóstico."

A Sociedade Brasileira de Diabetes (SBD) e também a Associação Americana de Diabetes (ADA) e Organização Mundial da Saúde (OMS) propõem quatro classes clínicas distintas: DM tipo 1 (DM1), DM tipo 2 (DM2), outros tipos específicos de DM e DM gestacional. No presente trabalho abordaremos o DM2.

De acordo com a SBD, o DM2 é a forma mais comum, sendo responsável por aproximadamente $90 \%$ dos casos de diabetes. Diferentemente do tipo 1, onde temos a destruição mediada pelo sistema imune das células $\beta$-pancreáticas, esse tipo da doença (2) é caracterizado por defeitos na ação e também secreção da insulina. Os fatores de risco para o desenvolvimento do DM2 incluem obesidade, sedentarismo, história familiar, idade avançada e história de diabetes gestacional. A ADA recomenda a triagem para DM2 em indivíduos com um ou mais fatores de risco.

Existe uma evidência crescente que relaciona a deficiência de vitamina $\mathrm{D}$ e a predisposição para o desenvolvimento do DM2, e também ao DM1, assim como a evolução de ambas as doenças. A National Health and Nutrition Examination Survey (NHANES III), avaliou 9.773 adultos maiores de 18 anos e mostrou uma associação entre concentrações de vitamina $\mathrm{D}$, homeostase de glicose e evolução do DM. Esse estudo observou uma relação inversa entre os valores de hemoglobina glicada (HbA1c) e as concentrações de $25(\mathrm{OH}) \mathrm{D}$ nas pessoas entre 35 e 74 anos sem história conhecida de DM. Os mecanismos biológicos subjacentes ainda não estão completamente elucidados.

As evidências atuais sugerem que a influência da 1,25-dihidroxicolecalciferol - 1,25(OH)2D3 na homeostase glicídica seja mediada por ações diretas e indiretas nas células $\beta$-pancreáticas, as quais expressam a enzima $1 \alpha$-hidroxilase e o receptor
VDR. Segundo Zierold et al. (2003) os prováveis mecanismos envolvidos no controle da síntese e secreção da insulina envolveriam a modulação do influxo e da reserva de $\mathrm{Ca} 2+$ no citosol, por mecanismos rápidos não genômicos do VDR na membrana das células beta pancreáticas, facilitando a clivagem da pró-insulina em insulina pelas endopeptidases cálcio-dependentes e estimulando a exocitose dos grânulos de insulina.

De acordo com Bland et al. (2004) o desenvolvimento de DM2 envolve alterações na função das células $\beta$-pancreáticas do pâncreas e resistência periférica à ação da insulina. A $25(\mathrm{OH})$ $\mathrm{D}$ pode atuar nesses mecanismos já que no tecido pancreático está presente o VDR e proteínas ligadoras de cálcio dependente de vitamina $\mathrm{D}$.

Como citado anteriormente, a vitamina $\mathrm{D}$ pode influenciar na resposta insulínica ao estímulo da glicose direta ou indiretamente. $\mathrm{O}$ efeito direto parece ser mediado pela ligação da 1,25(OH)2D3 ao VDR da célula $\beta$; alternativamente, a ativação da vitamina $\mathrm{D}$ pode acontecer no interior das células $\beta$ pela enzima $1 \alpha$-hidroxilase, expressa nessas células.

$\mathrm{O}$ efeito indireto é mediado pelo fluxo de $\mathrm{Ca} 2+$ intra e extracelular nas células $\beta$. Através de estudo experimental, Holecki, Zahorska-Markiewicz e Wiecek (2008) demonstrou que o aumento nos níveis séricos de $1,25(\mathrm{OH}) 2 \mathrm{D} 3$ e no PTH induz maior influxo de cálcio para o interior das células. Sendo a secreção de insulina um processo cálcio dependente mediado pela 1,25(OH)2D3 e pelo PTH, o aumento nas concentrações dessas substâncias, devido à insuficiência de $25(\mathrm{OH}) \mathrm{D}$, pode reduzir a capacidade secretora dessas células. Adicionalmente, a deficiência de $25(\mathrm{OH}) \mathrm{D}$ parece dificultar a capacidade das células $\beta$ na conversão da pró-insulina à insulina.

Outra discussão importante onde se verifica influência da vitamina D na resposta insulínica, diz respeito à calbindina, que é uma proteína ligante de cálcio, vitamina $\mathrm{D}$ dependente, que há muito se sabe está presente no pâncreas de várias espécies, 
inclusive em humanos. De acordo com Sooy et al. (1999), a calbindina é um importante regulador da produção de insulina, através da regulação da concentração de cálcio na célula $\beta$.

Calle, Maestro e Garcia-Arencibia (2008) realizaram um estudo com animais que teve por objetivo examinar os efeitos do tratamento com 1,25(OH)2D (150 UI/Kg, ou 3,75 $\mu \mathrm{g} / \mathrm{Kg}$ ), uma vez ao dia, durante quinze dias, em ratos não diabéticos e também em ratos com diabetes induzida por uma única injeção de estreptozotocina $(65 \mathrm{mg} / \mathrm{Kg})$. O tratamento com $1,25(\mathrm{OH}) 2 \mathrm{D}$ para os ratos com diabetes induzida por estreptozotocina não foi capaz de corrigir a hiperglicemia, hipoinsulinemia, glicosúria ou cetonemia ocasionadas pela diabetes, entretanto houve uma reversão parcial da superexpressão do gene do receptor de insulina no fígado e no tecido adiposo, sem alterar a expressão normal deste gene no rim. Estes efeitos foram acompanhados por uma normalização do número de receptores insulínicos, não alterando a afinidade pelo receptor, mas sim com uma melhoria da resposta insulínica para o transporte de glicose em adipócitos nestes animais diabéticos.

Estudos observacionais demonstram que a alta ingestão de vitamina $\mathrm{D}$ e/ou cálcio, e produtos lácteos foram inversamente associados com concentrações de glicose e insulina e incidência de DM2. Utilizando dados do Women's Health Study (37.183 mulheres sem história de DM2, doença cardiovascular e/ou câncer), Liu et al. (2005) relataram em um estudo prospectivo, que a ingestão total de vitamina D acima de $13 \mathrm{mg}$ / dia, aproximadamente, estava associado com um menor risco de incidência de DM2 em comparação com uma ingestão total diária menor do que $4 \mathrm{mg}$.

Corroborando estes dados, Pittas et al. (2006) também realizaram um estudo prospectivo que tinha por objetivo avaliar a associação entre ingestão de vitamina D e cálcio e o risco de desenvolver DM2. Acompanharam 83.779 mulheres participantes do Nurses' Health Study que não possuíam história de diabetes, doença cardiovascular, ou câncer no início do estudo, para verificar a incidência de DM2. A vitamina D e cálcio provenientes da dieta e também de suplementação foram avaliados a cada 2-4 anos, e durante 20 anos de seguimento foram documentados 4.843 casos incidentes de DM2. Após tratar os dados para eliminar possíveis interferências, os autores não encontraram associação entre a ingestão total de vitamina $\mathrm{D}$ e o DM2, porém o risco relativo (RR) para DM2 foi de 0,87 (IC 95\% 0,75-1,00; P = 0,04) comparando a mais alta com a mais baixa categoria de ingestão de vitamina D provenientes de suplementos. Os resultados deste estudo sugerem que a vitamina $\mathrm{D}$ e cálcio associado podem exercer um papel benéfico para redução do risco de DM2.

Estudos clínicos randomizados controlados (RCT) são relativamente raros, e os que são encontrados geralmente utilizam pacientes com DM2 e outras doenças de base, como obesidade por exemplo. Exemplo disso é o estudo de Nagpal et al. que em 2009 realizaram um estudo duplo cego, randomizado, placebo-controlado, com o objetivo de determinar o efeito da suplementação à curto prazo de vitamina $\mathrm{D}$, na sensibilidade à insulina em homens (100 voluntários do sexo masculino, com idade $\geq 35$ anos) aparentemente saudáveis, de meia idade, com obesidade central. Em um grupo, cada indivíduo recebeu três doses (120.000 UI) de vitamina D, quinzenalmente. No grupo controle foi administrado placebo com a mesma periodicidade. A sensibilidade hepática à insulina no jejum, HOMA-IR, índice quantitativo de verificação da sensibilidade à insulina (HOMA2), sensibilidade insulínica pós-prandial (oral glucose insulin sensitivity, OGIS), a secreção de insulina (HOMA\%B, HOMA2-\%B), perfil lipídico e pressão arterial foram mensurados no início do estudo e após 6 semanas de seguimento. Setenta e um indivíduos completaram o estudo (35 do grupo suplementado e 36 do grupo controle) e os autores relataram aumento significativo da OGIS no grupo suplementado. Os demais parâmetros avaliados não 
sofreram alteração. O estudo indicou que a vitamina $\mathrm{D}$ melhora a sensibilidade à insulina pós-prandial em homens (com obesidade central, porém não diabéticos) propensos a ter resistência insulínica.

Em outro RCT realizado por Von Hurst (2010), dessa vez com um período de seguimento mais longo, foi constatado que a suplementação com 100 mcg de vitamina D (4.000 UI) diariamente, durante 6 meses, foi capaz de melhorar a sensibilidade / resistência insulínica e a concentração de insulina em jejum em mulheres do sul asiático (Auckland, Nova Zelândia, $n=81$ ), com idade entre 23 a 68 anos, que apresentavam resistência à insulina (HOMA-IR $\geq 1,93$ ) e deficiência de vitamina $\mathrm{D}(<$ $50 \mathrm{nmol} / \mathrm{L}$ ). A melhoria do status de vitamina $\mathrm{D}$ em mulheres resistentes à insulina resultou na melhoria da resistência a insulina e de sensibilidade, mas nenhuma alteração significativa foi encontrada na secreção de insulina. As concentrações ótimas de vitamina $\mathrm{D}$ para a redução da resistência a insulina foram de 80 a $119 \mathrm{nmol} / \mathrm{L}$.

Em contraste, Jorde e Figenschau (2009) concluíram um estudo no qual participaram 36 pacientes diabéticos (DM2), porém sem deficiência de vitamina $\mathrm{D}$, onde se verificou que a suplementação com essa vitamina (400.000 UI, semanalmente) durante 6 meses, não apresentou efeito significativo sobre o metabolismo da glicose nesses pacientes.

Em síntese, os resultados dos ensaios sobre os efeitos que a vitamina $\mathrm{D}$ pode exercer no DM2 mostram que a deficiência de $25(\mathrm{OH}) \mathrm{D}$ altera a produção e a secreção da insulina, tanto em animais como em humanos. Outrossim, a deficiência dessa vitamina parece conduzir à intolerância a glicose e a secreção insulínica deletéria, contribuindo para o maior risco de desenvolvimento de DM2. No entanto, sabemos que o diabetes mellitus é uma doença multifatorial, cuja prevenção exige alterações no estilo de vida, como por exemplo, dieta equilibrada e a prática regular de exercícios físicos. Estes achados que evidenciam a participação da vitamina D nos processos de secreção e ação da insulina e também no controle glicêmico, sugestionam que essa vitamina deve ser mais um fator considerado tanto na prevenção como no tratamento do DM.

\section{Vitamina D e Hipertensão Arterial Sistêmica}

A hipertensão arterial sistêmica (HAS) é uma condição clínica multifatorial que se caracteriza por níveis elevados e sustentados de pressão arterial (PA). Essa condição pode configurar alterações estruturais e funcionais nos órgãos-alvo, como o coração, encéfalo, rins e vasos sanguíneos e também pode levar a alterações metabólicas, aumentando o risco de eventos cardiovasculares fatais e não fatais.

Segundo a Sociedade Brasileira de Cardiologia, a HAS tem alta prevalência e baixas taxas de controle, e é considerada um dos principais fatores de risco modificáveis e um dos mais importantes problemas de saúde pública. A mortalidade por doença cardiovascular (DCV) aumenta progressivamente com a elevação da PA a partir de 115/75 mmHg de forma linear, contínua e independente.

De acordo com Passos, Assis e Barreto (2006, p. 36) no Brasil, as doenças cardiovasculares são responsáveis por $33 \%$ dos óbitos com causas conhecidas. A hipertensão é a mais prevalente de todas as DCV, afetando mais de 36 milhões de brasileiros adultos, além disso, é considerada o maior fator de risco para lesões cardíacas e cerebrovasculares e a $3^{\text {a }}$ causa de invalidez. A hipertensão provavelmente está envolvida em $50 \%$ das mortes causadas por DCV.

A hipertensão ocorre principalmente pela ativação inadequada do sistema renina-angiotensina e existem vários estudos que indicam que os níveis séricos de 1,25(OH)2D estão inversamente associados à pressão arterial ou à atividade da renina plasmática em normotensos e hipertensos (SCHUCH et al., 2009).

A renina, uma protease, é sintetizada principalmente nas células justaglomerulares dos rins e catalisa a conversão do angiotênsinogênio, produzido pelo fígado, em angiotensina I, que é 
clivada em angiotensina II pela ação da enzima conversora de angiotensina (ECA), presente no endotélio vascular de vários órgãos. $\mathrm{O}$ aumento nos níveis de angiotensina II eleva a pressão arterial de duas formas: 1) Aumentando a resistência vascular periférica, por ser uma substância vasoconstritora potente; 2) Estimulando a secreção de aldosterona, que por sua vez, aumenta a reabsorção renal de sódio, culminando com o aumento da pressão arterial.

Várias células que compõem o sistema cardiovascular expressam a CYP27B1, enzima de conversão da 25(OH)D em 1,25(OH)2D e/ ou o VDR, como as células musculares lisas e endoteliais dos vasos sanguíneos, miócitos, e as células justaglomerulares do néfron (produtoras de renina). A 1,25(OH)2D participa do controle da função cardíaca e da pressão arterial por meio da regulação do crescimento das células musculares lisas, do grau de contratilidade miocárdica e da inibição da renina, interferindo na dinâmica do sistema renina-angiotensina-aldosterona. (Li et al., 2004)

Holick (2007) afirma que a vitamina D tem a capacidade de afetar o sistema renina-angiotensina, que é o principal sistema responsável pelo controle da PA, além de exercer efeitos benéficos sobre as células do tecido liso vascular, o endotélio e os cardiomiócitos. A ação da 1,25(OH)2D sobre a expressão gênica ocorre por meio do VDR presente nos tecidos, como citado anteriormente. Kong et al., em 2008, demonstraram que a supressão da expressão de renina pela $1,25(\mathrm{OH}) 2 \mathrm{D}$ in vivo é independente do PTH e do cálcio.

Evidências em estudos observacionais apoiam fortemente a existência de relação entre pressão arterial e vitamina D, Scragg et al. (2007) analisando dados da $3^{\mathrm{a}}$ National Health and Nutrition Examination Survey (NHANESIII), constataram que concentrações séricas elevadas de $25(\mathrm{OH})$ $\mathrm{D}$ estavam associadas com baixa prevalência de hipertensão arterial, nos indivíduos participantes.
Reforçando essas afirmações, Li et al. (2004) realizaram um experimento com camundongos onde os animais knockout para o receptor VDR tiveram os níveis de renina e angiotensina II aumentados e desenvolveram hipertensão arterial. Um fenótipo semelhante ocorre com animais que tiveram o gene da $1 \alpha$-hidroxilase silenciado. Em outro estudo Freundlich, Quiroz e Zhang (2008) relataram que a injeção de análogos da 1,25(OH)2D3 em camundongos ocasionou a supressão da produção de renina in vivo e regulou negativamente a expressão do gene do angiotensinogênio.

Em humanos, dados do Intersalt Study (INTERSALT COOPERATIVE RESEARCH GROUP, 1988), trabalho importante acerca dos fatores de risco e controle da hipertensão, com mais de 10.000 participantes de diversos países, demonstraram que a pressão arterial sistólica e diastólica foram associadas positivamente à distância do equador, e com isso demonstraram que a exposição solar e, supostamente, a menor concentração de 25(OH)D estariam relacionadas à PA. Somado a isso, Krause et al. (1998) realizaram um estudo avaliando indivíduos hipertensos que foram expostos à radiação UVA e UVB, semelhante à luz solar, em uma câmara de bronzeamento, 3 vezes por semana durante 3 meses. O grupo controle foi submetido ao mesmo procedimento, porém utilizando somente radiação do tipo UVA. Foi observado um aumento significativo (180\%) nos níveis séricos de 25(OH)D e uma diminuição $(6 \mathrm{mmHg})$ na pressão sistólica e diastólica no grupo submetido aos dois tipos de radiação.

Por outro lado, para elucidar a questão do possível efeito preventivo que a suplementação de vitamina $\mathrm{D}$ poderia exercer sobre o desenvolvimento da hipertensão arterial, Forman et at. (2005) avaliaram três grandes estudos de coorte prospectivos, sobre ingestão de vitamina D e o risco de desenvolver hipertensão, compreendendo mais de 200.000 participantes do Nurses' Health Studies (NHS I, $n=77.436$ e NHS II, $n=93.803$ ) $e$ 
do Health Professionals Follow-up Study (HPFS, $n=38.074)$. Cada grupo foi acompanhado durante 8 anos ou mais e foi constatado que a ingestão de vitamina $\mathrm{D}$ não estava associada com o risco de desenvolvimento de hipertensão. As estimativas de risco relativo multivariável para o mais alto quintil em comparação com o quintil mais baixo de ingestão de vitamina D foram $0,98(0,93$ - 1,04) em NHS I, 1,13 (0,99 - 1,29) em NHS II, e 1,03 $(0,93$ - 1,15) em HPFS. Quando se comparavam os participantes que consumiram $\geq 1600$ UI a $<400$ UI por dia com aqueles que consumiram $\geq 1000 \mathrm{UI}$ a $<200$ UI por dia, não foi encontrada associação. Assim, concluíram que o maior consumo de vitamina D não esteve associado com um menor risco de incidência de hipertensão.

Neves et al. (2012) realizaram um estudo transversal comparando as concentrações de $25(\mathrm{OH}) \mathrm{D}$ e fatores relacionados à prevalência da hipovitaminose D, com a PA em 91 idosos hipertensos. Foram comparados dados bioquímicos, antropométricos, dietéticos, tipo de pele, exposição solar e PA. A prevalência da inadequação da vitamina $\mathrm{D}(<29 \mathrm{ng} / \mathrm{mL})$ foi de $33 \%$ e a concentração sérica da 25(OH)D associou-se inversamente com a pressão arterial sistólica e positivamente com a frequência semanal do consumo de peixes, concluindo que a prevalência da inadequação das concentrações de vitamina $\mathrm{D}$ foi elevada e relacionada com a maior PA em idosos, estando por outro lado, o maior consumo semanal de peixe ligado a maiores concentrações de 25(OH)D.

Em outro estudo epidemiológico conduzido por Forman et al. (2008) realizado com 1.484 mulheres participantes do Nurses' Health Study, divididas em 742 casos que desenvolveram hipertensão e 742 controles que não desenvolveram, foi observado que a concentração de $25(\mathrm{OH}) \mathrm{D}$ era menor nos casos $(25,6 \mathrm{ng} / \mathrm{mL})$ do que nos controles $(27,3 \mathrm{ng} / \mathrm{mL} ; \mathrm{p}$ $=0,001)$. Aquelas mulheres que se encontravam no menor quartil de vitamina D apresentaram maior risco para desenvolvimento da hipertensão do que aquelas no maior quartil (razão de chance ajustada de 1,66; IC95\% = 1,11-2,48; $\mathrm{p}=0,01)$.

Lind et al. em 1987 já realizavam estudo de suplementação, duplo-cego placebo-controlado, onde observaram a redução na pressão arterial de 39 indivíduos hipertensos com a suplementação de vitamina D. Adicionalmente, Pfeifer M. et al. (2001) relataram que essa redução também foi constatada em mulheres idosas suplementadas com cálcio e vitamina $\mathrm{D}$, durante oito semanas.

Mesmo diante dos resultados dos estudos que vêm mostrando essa relação inversa entre vitamina D e pressão arterial, os autores ainda alertam que resultados de estudos randomizados de suplementação de vitamina D (com colecalciferol ou ergocalciferol) para normalizar a pressão arterial em pacientes hipertensos são inconsistentes, possivelmente em virtude da variabilidade na população do estudo, tamanho da amostragem, dose e duração da intervenção (NEVES et al., 2012).

Assim sendo, é importante avaliar melhor os efeitos da suplementação de 25(OH)D em estudos de base populacional, desse modo será possível estabelecer o status de vitamina D necessário para prover benefícios no que tange a hipertensão arterial, que é um problema de saúde pública e, uma vez confirmando o papel benéfico da vitamina $D$ sobre os níveis pressóricos, esta pode ser vista como um coadjuvante no tratamento de hipertensos.

\section{Vitamina D e Obesidade}

A obesidade é uma doença crônica não transmissível (DCNT) caracterizada pelo excesso de gordura corporal e resultante do desequilíbrio prolongado entre o consumo alimentar e o gasto energético (PEIXOTO et al., 2006, p. 463). Atualmente é um grave problema de saúde pública, atingindo proporções "epidêmicas" tanto em países desenvolvidos como em nações e regiões em desenvolvimento, com grande impacto sobre o padrão de morbidade de populações adultas. Enquanto a etiologia da obesidade é multifatorial, os principais 
fatores para seu desenvolvimento na atual epidemia dessa doença são de origem alimentar.

Segundo a Organização Mundial da Saúde (OMS, 2003), ela pertence ao grupo das Doenças Crônicas Não Transmissíveis - DCNT que abarca ainda diabetes mellitus, doenças cardiovasculares, hipertensão arterial, infarto do miocárdio e alguns tipos de cânceres.

Estimativas da OMS apontam as DCNT como responsáveis por $58,5 \%$ de todas as mortes e por $45,9 \%$ da carga global da morbidade adulta em todo o mundo. Ainda de acordo com essa organização, em 2008 cerca de 1,5 bilhão de adultos apresentavam sobrepeso e, destes, mais de 200 milhões de homens e aproximadamente 300 milhões de mulheres eram obesos. Se compararmos com dados de 1980, o aumento na prevalência de obesidade foi de mais de duas vezes, e as projeções para os próximos anos indicam que, em 2030, um número próximo a 3,3 bilhões de pessoas, ou $57,8 \%$ da população adulta mundial, terá sobrepeso ou obesidade. "No Brasil, cerca de metade da população adulta apresenta excesso de peso, enquanto $12,5 \%$ dos homens e $16,9 \%$ das mulheres apresentam obesidade." (IBGE, 2010).

A obesidade abdominal ou andróide, definida pelo aumento de tecido adiposo na região abdominal, é considerada um fator de risco independente para diversas morbidades, representando risco diferenciado quando comparada com outras formas de distribuição de gordura corporal. Esse tipo de obesidade predispõe o indivíduo a uma série de fatores de risco cardiovasculares por associar-se com grande frequência a condições como dislipidemias, hipertensão arterial, resistência à insulina e diabetes mellitus que favorecem a ocorrência de eventos cardiovasculares agudos, particularmente os coronarianos.

Vários estudos relatam que em indivíduos obesos é frequente a observação de níveis séricos reduzidos de $25(\mathrm{OH}) \mathrm{D}$. Investiga-se que a insuficiência de vitamina $\mathrm{D}$ em obesos pode ser um dos fatores que desencadeie o acúmulo de gordura corporal, e não seja apenas consequência de menor exposição solar por parte deste público devido ao baixo nível de atividade física e menor mobilidade.

Por outro lado, sabe-se que os macrófagos estão envolvidos na conversão periférica da $25(\mathrm{OH}) \mathrm{D}$ no metabólito ativo 1,25(OH)2D (BIKLE, 2009) e na obesidade há a expansão do tecido adiposo com o aumento da acumulação de macrófagos neste tecido, o que poderia facilitar a hidroxilação da 25(OH)D. Para explicar isso, os autores sugerem que níveis aumentados de 1,25(OH)2D seriam responsáveis pelo feedback negativo à síntese hepática da $25(\mathrm{OH})$ $\mathrm{D}$, o que também explicaria a baixa disponibilidade desta em obesos, porém são necessários mais estudos para evidenciar essa hipótese.

De acordo com Blum et al. (2008), o tecido adiposo funciona como um depósito de 25(OH)D e seus metabólitos, devido às suas propriedades lipofílicas, diminuindo a sua biodisponibilidade e acionando o hipotálamo para desenvolver uma cascata de reações que culmina no aumento da sensação de fome e diminuição do gasto energético. Esta situação gera aumento nos níveis de PTH, consequente diminuição da sensibilidade à insulina e aumento desproporcional na concentração de cálcio intracelular.

Em estudos epidemiológicos, de base populacional foi demonstrada uma correlação negativa entre gordura corporal total e concentração sérica de $25(\mathrm{OH}) \mathrm{D}$, que se manteve significativa após o ajuste para idade, estação do ano, ingestão de vitamina D e raça (SNIJDER, 2005).

Reforçando esses dados Su e Zemel (2008) confirmaram essa estreita relação através da determinação dos níveis de $25(\mathrm{OH}) \mathrm{D}$ no soro e no tecido adiposo de pacientes obesos submetidos à cirurgia gástrica by-pass, mostrando uma relação inversa dos níveis de $25(\mathrm{OH}) \mathrm{D}$ com o peso corporal e adiposidade.

Adicionalmente, Snijder et al. (2005) realizaram um estudo chamado Longitudinal Aging Study Amsterdam (LASA) e verificaram que a baixa 
concentração de $25(\mathrm{OH}) \mathrm{D}$ e a maior concentração de PTH estavam associados fortemente com a soma das dobras cutâneas e o percentual de gordura corporal dos indivíduos participantes.

O tecido adiposo, além de ser considerado estoque de energia, funciona como uma glândula endócrina que pode secretar uma variedade de proteínas bioativas denominadas adipocinas, dentre elas podese citar a adiponectina, a leptina, a grelina, o TNF- $\alpha$, a IL-6 e a proteína quimiotática de monócitos-1 (MCP-1), essas adipocinas estão diretamente envolvidas no processo inflamatório. Ainda há poucos estudos que analisaram o possível papel que a vitamina $\mathrm{D}$ pode desempenhar na modulação da produção de adipocinas. Camundongos knockout para o receptor VDR ou para a enzima $1 \alpha$-hidroxilase têm reduzidos níveis de leptina no soro, hormônio peptídico produzido pelos adipócitos e relacionado principalmente ao controle do apetite (NARVAEZ et al., 2009).

A vitamina D também está envolvida na adipogênese, de acordo com Schuch et al. (2009, p. 629),

Recentes estudos feitos com modelos de culturas de pré-adipócitos de camundongos levantaram a hipótese de que o papel da $25(\mathrm{OH}) \mathrm{D}$ na inibição da adipogênese é mediado em nível molecular por meio da inibição do VDR e da expressão do reguladormestre da adipogênese, o receptor ativador de proliferação de peroxissomos $\gamma(\operatorname{PPAR} \gamma)$, reduzindo, consequentemente, sua atividade.

Blumberg et al. (2006) realizaram um estudo utilizando pré-adipócitos (3T3-L15B2), que foram tratados com a 1,25(OH)2D e constataram que houve a inibição parcial da formação endógena de ligantes de PPARy, o componente chave da adipogênese. Desse modo, com a redução da expressão desse componente com consequente diminuição da sua atividade na presença da $1,25(\mathrm{OH}) 2 \mathrm{D}$, houve inibição da adipogênese. Porém se trata de um experimento in vitro e pesquisas adicionais são necessárias para entender a diminuição de PPARy na presença de $1,25(\mathrm{OH}) 2 \mathrm{D}$ e investigar a extensão da interação entre VDR e PPARy em pré adipócitos in vivo. Alguns autores sugerem ainda que a
1,25(OH)2D pode induzir à apoptose mediada por cálcio nos adipócitos, levando a uma diminuição do tecido adiposo (SERGEEV et al., 2009).

Semelhante ao que foi descrito no trabalho anterior, realizado por Blumberg et. al (2006) utilizando préadipócitos (3T3-L15B2), foi constatado também nesse estudo que a $1,25(\mathrm{OH}) 2 \mathrm{D}$ inibe estágios inicias de diferenciação de pré-adipócitos ( $7^{\circ}$ dia), porém no $14^{\circ}$ dia, a influência da $1,25(\mathrm{OH}) 2 \mathrm{D}$ diminuiu e a diferenciação ocorreu igualmente entre os poços, tratados ou não com vitamina $\mathrm{D}$. Curiosamente, a 25(OH)D funciona igualmente bem para inibir a diferenciação dos pré-adipócitos no $7^{\circ}$ dia, o que indica que essas células tem a capacidade de bioativar a $25(\mathrm{OH}) \mathrm{D}$ em $1,25(\mathrm{OH}) 2 \mathrm{D}$ e sinalizar via VDR para regular a diferenciação em adipócitos, semelhante ao tratamento direto com 1,25(OH)2D. Embora o tratamento com $1,25(\mathrm{OH}) 2 \mathrm{D}$ ou $25(\mathrm{OH})$ D tenha mostrado uma tendência na redução da diferenciação dos adipócitos após 14 dias, foi apenas no $7^{\circ}$ dia que foi detectado uma diferença significativa desse fenômeno. Desse modo podese concluir que a vitamina $\mathrm{D}$ pode inibir o início do processo de diferenciação em adipócitos, mais não bloqueia completamente este processo, em préadipócitos humanos primários.

Não obstante, os dados sobre a ação in vivo da vitamina $\mathrm{D}$ e sua relação com a adipogênese ainda são escassos e mais estudos são necessários.

$\mathrm{Na}$ prevenção da obesidade utilizando a suplementação com essa vitamina, Caan et al. (2007) realizaram a análise dos dados de um estudo chamado Women's Health Initiative (WHI) que englobou 36.282 mulheres na pós menopausa, com idade entre 50 e 79 anos, e verificaram menor ganho de peso durante o uso de suplementação com 1.000 $\mathrm{mg}$ de cálcio e 400UI de colecalciferol, quando comparadas com o grupo controle.

Quando se trata de indivíduos já obesos, Sneve, Figenschau e Jorde (2008) observaram em um estudo duplo cego placebo-controlado, que a suplementação de colecalciferol não ocasionou 
mudanças significativas em peso, relação cinturaquadril e percentual de gordura corporal. O estudo utilizou 445 indivíduos de 21 a 70 anos e IMC entre 28 e $47 \mathrm{~kg} / \mathrm{m} 2$, que foram divididos em 3 grupos: a) 20.000UI de colecalciferol duas vezes por semana; b) 20.000UI de colecalciferol uma vez por semana e placebo uma vez por semana; c) Placebo duas vezes por semana. Todos os indivíduos receberam 500mg de cálcio. Os resultados demonstraram aumento significativo na concentração sérica de 25(OH)D e redução do PTH.

Tomados em conjunto, a vitamina $\mathrm{D}$, ou a vitamina D somada a suplementação de cálcio parecem ter um efeito na restrição do ganho de peso na população em geral, mais os seus efeitos na redução de peso de indivíduos já obesos podem ser limitados. Mai et al. (2011) sugerem haver um ciclo prejudicial ( $\downarrow$ vitamina D obesidade $\downarrow$ vitamina D) que complica a prevenção da obesidade e os esforços de tratamento. Essa possibilidade merece pesquisas futuras.

\section{Conclusão}

A deficiência de vitamina $\mathrm{D}$ e suas consequências constituem um problema de saúde pública mundial. Nos países subdesenvolvidos, a hipovitaminose D ainda não recebe a devida atenção enquanto problema de saúde. Como podemos observar nesta revisão, vários estudos têm mostrado que a vitamina $\mathrm{D}$ pode desempenhar um papel importante em muitos mecanismos bioquímicos para além do metabolismo ósseo e do cálcio. Apesar das evidências encontradas (grande parte em estudos transversais), dados de estudos prospectivos e ensaios clínicos randomizados ainda são escassos, porém necessários para demonstrar os efeitos clínicos e metabólicos das variações na concentração da $25(\mathrm{OH}) \mathrm{D}$ na evolução clínica de diversas doenças, como as que discutimos neste trabalho, e também para estabelecer o momento e necessidade de começar uma terapia utilizando vitamina $\mathrm{D}$, bem como para determinar a dose, via e duração da administração. Vale ressaltar que ainda não há consenso na literatura médica sobre os pontos de corte que caracterizam suficiência, insuficiência ou deficiência de vitamina D no organismo. A investigação da adequação do status desta vitamina deve ser objeto de pesquisas futuras, além disso, a busca de análogos sintéticos de vitamina D sem atividade calcêmica também pode ser promissora para a prevenção e tratamento das doenças endocrinometabólicas.

\section{Referências}

AL-DAGHRI, N. M.; ALKHARFYA, K. M.; ALSALEHB, Y.; AL-ATTASA, O. S.; ALOKAILA, M. S.; AL-OTHMANB, A.; MOHARRAMG, O.; EL-KHOLIEH, E.; SABICOA, S.; KUMARI, S.; CHROUSOSA, G. P. Modest reversal of metabolic syndrome manifestations with vitamin D status correction: a 12-month prospective study. Metabolism Journal, Amsterdam, v. 61, p. 661-666, 2012.

BIKLE, D. D. Extra renal synthesis of 1, 25-dihydroxyvitamin D and its health implications. Clinical Reviews in Bone and Mineral Metabolism, Totowa, v. 7, p. 114-125, 2009.

BLAND, R.; MARKOVIC, D.; HILLS, C. E.; HUGHES, S. V.; CHAN, S. L.; SQUIRES, P. E. Expression of 25-hydroxivitamin D3-alphahydroxylase in pancreatic islets. The Journal of Steroid Biochemistry and Molecular Biology, Oxford, v. 89-90, p. 121-125, 2004.

BLUM, M.; DOLNIKOWSKI, G.; SEYOUM, E.; HARRIS, S. S.; BOOTH, S. L.; PETERSON, J.; SALTZMAN, E.; DAWSON-HUGHES, B. Vitamin D 3 in fat tissue. Endocrine, Totowa, v. 33, p. 90-94, 2008.

BLUMBERG, J. M.; TZAMELI, I.; ASTAPOVA, I.; LAM, F. S.; FLIER, J. S.; HOLLENBERG, A. N. Complex role of the vitamin $\mathrm{D}$ receptor and its ligand in adipogenesis in 3T3-L1 cells. The Journal of Biological Chemistry, Baltimore, v. 281, p. 11205-11213, 2006. 
IBGE. Pesquisa de orçamentos familiares: 20082009. Rio de Janeiro, 2010.

CAAN, B.; NEUHOUSER, M.; ARAGAKI, A.; LEWIS, C. B.; JACKSON, R.; LEBOFF, M. S. Calcium plus vitamin D supplementation and the risk of postmenopausal weight gain. Archives of Internal Medicine, Chicago, v. 167, n. 9, p. 893-902, 2007.

CALLE,C.;MAESTRO,B.; GARCIA-ARENCIBIA, M. Genomic actions of 1,25-dihydroxyvitamin D3 on insulin receptor gene expression, insulin receptor number and insulin activity in the kidney, liver and adipose tissue of streptozotocin- induced diabetic rats. BMC Molecular Biology, London, v. 9, n. 65, 2008.

FORD, E. S; AJANI, U. A; MCGUIRE, L. C.; LIU, S. Concentrations of serum vitamin D and the metabolic syndrome among U.S. adults. Diabetes Care, Alexandria, v. 28, p. 1228-1230, 2005.

FORMAN, J. P.; BISCHOFF-FERRARI, H. A.; WILLETT, W. C.; STAMPFER, M. J.; CURHAN, G. C. Vitamin D intake and risk of incident hypertension: results from three large prospective cohort studies. Hypertension, Dallas, v. 46, p. 676-682, 2005.

FORMAN, J. P.; CURHAN, G. C.; TAYLOR, E. $\mathrm{N}$. Plasma 25-hydroxyvitamin D levels and risk of incident hypertension among young women. Hypertension, Dallas, v. 52, p. 828-32, 2008.

FREUNDLICH, M.; QUIROZ, Y.; ZHANG, Z. Suppression of renin-angiotensin gene expression in the kidney by paricalcitol. Kidney International, New York, v. 74, p. 1394-402, 2008.

GRUNDY, S. M.; CLEEMAN, J. I.; DANIELS, S. R.; DONATO, K. A.; ECKERL, R. H.; FRANKLIN, B. A. Diagnosis and management of the metabolic syndrome: an American Heart Association/ National Heart, Lung, and Blood Institute Scientific Statement. Circulation, Dallas, v. 112, n. 17, p. 2735-2752, 2005.

HOLECKI, M.; ZAHORSKA-MARKIEWICZ, B.; WIECEK, A. Influence of calcium and vitamin D supplementation on weight and fat loss in obese women. Obesity Facts, Basel, v. 1, p. 274-279, 2008.
HOLICK, M. F. Vitamin D: deficiency. New England Journal of Medicine, Chicago, v. 357, p. 266-281, 2007.

HOLICK, M. F. Vitamin D: importance in the prevention of cancers, type 1 diabetes, heart disease and osteoporosis. The American Journal of Clinical Nutrition, Bethesda, v. 79, n. 5, p. 362-371, 2004.

INSTITUTE OF MEDICINE - IOM. Dietary reference intakes for calcium and vitamin D. National Academies Press. Washington, DC. 2011.

INTERSALT COOPERATIVERESEARCH GROUP. Intersalt: an international study of electrolyte excretion and blood pressure: results for 24 hour urinary sodium and potassium excretion. British Medical Journal, London, v. 297, p. 319-28, 1988.

JORDE, R.; FIGENSCHAU, Y. Supplementation with cholecalciferol does not improve glycaemic control in diabetic subjects with normal serum 25-hydroxyvitamin D levels. European Journal of Nutrition, Kuopio, v. 48, p. 349-354, 2009.

KAYANIYIL, S.; VIETH, R.; HARRIS, S. B.; RETNAKARAN, R.; KNIGHT, J. A.; GERSTEIN, H. C.; PERKINS, B. A.; ZINMAN, B.; HANLEY, A. J. Association of $25(\mathrm{OH}) \mathrm{D}$ and $\mathrm{PTH}$ with metabolic syndrome and its traditional and nontraditional components. The Journal of Clinical Endocrinology and Metabolism, Springfield, v. 96, p. 168-175, 2011.

KONG, J.; QIAO, G.; ZHANG, Z.; LIU, S. Q.; LI, Y. C. Targeted vitamin $D$ receptor expression in juxtaglomerular cells suppresses renin expression independent of parathyroid hormone and calcium. Kidney International, New York, v. 74, p. 1577-1581, 2008.

KRAUSE, R.; BÜHRING, M.; HOPFENMÜLLER, W.; HOLICK, M. F.; SHARMA, A. M. Ultraviolet B and blood pressure. Lancet, London, v. 352, p. 709710, 1998.

LI, Y. C.; QIAO, G.; USKOKOVIC, M.; XIANG, W.; ZHENG, W.; KONG, J. Vitamin D: a negative endocrine regulator of the renin-angiotensin system and blood pressure. The Journal of Steroid Biochemistry and Molecular Biology, Oxford, v. 89, p. 387-92, 2004. 
LIND, L.; WENGLE, B.; LJUNGHALL, S. Blood pressure is lowered by vitamin D (alphacalcidol) during long-term treatment of patients with intermittent hypercalcemia: A double blind, placebo-controlled study. Acta Medica Scandinavica, Sweden, v. 222, p. 423-427, 1987.

LIU, S.; SONG, Y.; FORD, E. S. Dietary calcium, vitamin $\mathrm{D}$, and the prevalence of metabolic syndrome in middle-aged and older U.S. women. Diabetes Care, Alexandria, v. 28, p. 2926-2932, 2005.

MAI, X. M.; CHEN, Y.; CAMARGO JUNIOR, C. A; LANGHAMMER, A. Cross-sectional and prospective cohort study of serum 25-hydroxyvitamin D level and obesity in adults the HUNT study. American Journal of Epidemiology, Cary, v. 175, n. 10, p. 1029-1036, 2012.

MALERBII, D. A.; FRANCO, L. J. Multicenter study of the prevalence of diabetes mellitus and impaired glucose tolerance in the urban Brazilian population aged 30-69 years. The Brazilian cooperative group on the study of diabetes prevalence. Diabetes Care, Alexandria, v. 15, sup. 1, p. 1509-1516, 1992.

MIÑAMBRES， I.; SÁNCHEZ-HERNÁNDEZ, J.; SÁNCHEZ-QUESADA, J. L.; RODRÍGUEZ, J.; LEIVA, A.; PÉREZ, A. The association of hypovitaminosis $\mathrm{D}$ with the metabolic syndrome is independent of the degree of obesity. International Scholarly Research Network Endocrinology, New York, v. 2012, p. 1-5, 2012.

NAGPAL, J.; PANDE, J. N.; BHARTIA, A. A double-blind, randomized, placebo-controlled trial of the short-term effect of vitamin D3 supplementation on insulin sensitivity in apparently healthy, middle-aged, centrally obese men. Diabetic Medicini, Oxford, v. 26, p. 19-27, 2009.

NARVAEZ, C. J.; MATTHEWS, D.; BROUN, E. Lean phenotype and resistance to diet-induced obesity in vitamin D receptor knockout mice correlates with induction of uncoupling protein-1 in white adipose tissue. Endocrinology, Bristol, v. 150 p. 651-661, 2009.
NEVES, J. P. R.; SILVA, A. S.; MORAIS, L. C. S. L.; DINIZ, A. S.; COSTA, M. J. C.; ASCIUTTI, L. S. R.; GONÇALVES, M. C. R. Hipovitaminose D em idosos hipertensos. Arquivos Brasileiros de Endocrinologia e Metabologia, São Paulo, v. 56/57, p. 415-422, 2012.

ORGANIZAÇÃO MUNDIAL DA SAÚDE (OMS). Cuidados inovadores para condições crônicas: componentes estruturais de ação. Brasília, 2003.

PASSOS, V. M. A.; ASSIS, T. D.; BARRETO, S. M. Hipertensão arterial no Brasil: estimativa de prevalência a partir de estudos de base populacional. Epidemiologia e Serviços de Saúde, Brasília, v. 15, p. 35-45, 2006.

PEIXOTO, M. R. G.; BENICIO, M. H. D.; LATORRE, M. R. D. O.; JARDIM, P. C. B. V. Circunferência da cintura e índice de massa corporal como preditores da hipertensão arterial. Arquivos Brasileiros de Cardiologia, Rio de Janeiro, v. 87, p. 462-70, 2006.

PFEIFER, M.; BEGEROW, B.; MINNE, H. W.; NACHTIGALL, D.; HANSEN, C. Effects of shortterm vitamin D3 and calcium supplementation on blood pressure and parathyroid hormone levels in elderly women. The Journal of Clinical Endocrinology and Metabolism, Springfield, v. 86, p. 1633-1637, 2001.

PITTAS, A. G.; DAWSON-HUGHES, B.; LI, T. Vitamin D and calcium intake in relation to type 2 diabetes in women. Diabetes Care, Alexandria, v. 29, p. 650-656, 2006.

SCHUCH, N. J.; GARCIA, V. C.; MARTINI, L. A. Vitamina D e doenças endocrinometabólicas. Arquivos Brasileiros de Endocrinologia e Metabologia, São Paulo, v. 53, p. 625-633, 2009.

SCRAGG, R.; SOWERS, M.; BELL, C. Serum 25-hydroxyvitamin $\mathrm{D}$, ethnicity, and blood pressure in the third National health and nutrition Examination Survey. American Journal of Hypertension, Oxford, v. 20, p. 713-719, 2007.

SERGEEV,I.N.;LI, S.;HO,C.T.Polymethoxyflavones activate $\mathrm{Ca} 2 \mathrm{p}$-dependent apoptotic targets in adipocytes. Journal of Agricultural and Food Chemistry, Washington, v. 57, p. 5771-5776, 2009. 
SNEVE, M.; FIGENSCHAU, Y.; JORDE, R. Supplementation with cholecalciferol does not result in weight reduction in overweight and obese subjects. European Journal of Endocrinology, Bristol, v. 159, p. 675-84, 2008.

SNIJDER, M. B.; VAN DAM, R. M.; VISSER, M.; DEEG, D. J.; DEKKER, J. M.; BOUTER, L. M. Adiposity in relation to vitamin $\mathrm{D}$ status and parathyroid hormone levels: a population-based study in older men and women. The Journal of Clinical Endocrinology and Metabolism, Springfield, v. 90, p. 4119-4123, 2005.

SOCIEDADE BRASILEIRA DE CARDIOLOGIAIV DIRETRIZES BRASILEIRAS DE HIPERTENSÃO ARTERIAL. Arq Bras Cardiol 2010; 95(1 supl.1): 1-51

SOCIEDADE BRASILEIRA DE DIABETES SBD. Tratamento e acompanhamento do diabetes mellitus: diretrizes da Sociedade Brasileira de Diabetes. São Paulo, 2006.

SOOY, K.; SCHERMERHORN, T.; NODA, M. Calbindin-D28k controls [Ca (2p)] i and insulin release. Evidence obtained from calbindin-D28k knockout mice and $\mathrm{b}$ cell lines. The Journal of Biological Chemistry, Baltimore, v. 274, p. 3434334349, 1999.

SU, X.; ZEMEL, M. B. 1alpha, 25-dihydroxyvitamin $\mathrm{D}$ and corticosteroid regulate adipocyte nuclear vitamin D receptor. International Journal of Obesity, London, v. 32, p. 1305-1311, 2008.

VON HURST, P. R.; STONEHOUSE, W.; COAD, J. Vitamin D supplementation reduces insulin resistance in South Asian women living in New Zealand who are insulin resistant and vitamin D deficient: a randomized, placebo controlled trial. The British Journal of Nutrition, Cambridge, v. 103, p. 549-555, 2010.

ZIEROLD, C.; MINGS, J. A.; DELUCA, H. F. Regulation of 25-hydroxyvitamin D3-24hydroxylase mRNA by 1, 25-dihydroxyvitamin D3 and parathyroid hormone. Journal of Cellular Biochemistry, New York, v. 88, p. 234-237, 2003 
